\title{
A Preventive Strategy of Voltage Stability based on Characteristic Analysis
}

\author{
Zhen $\mathrm{Hu}^{1, *}$, Ding Wang ${ }^{1}$, Yangwu Shen ${ }^{1}$, Daojun $\mathrm{Chen}^{1}$, and Yongyan Liu ${ }^{1}$ \\ ${ }^{1}$ State Grid Hunan Electric Power Company Limited Research Institute, Power grid technology center, 410082, 79 Shuidian Street, \\ Yuhua District, Changsha City, Hunan Province, China
}

\begin{abstract}
The purpose of voltage stability prevention and control strategy is to minimize the impact of expected accidents on the whole power grid while ensuring safe power supply. In this paper, it is expected that the accident has a large impact on the system voltage and makes the voltage margin of each node low. in order to not only adjust the node voltage, but also reduce the disturbance impact, a voltage stability prevention and control strategy is proposed based on the characteristic analysis.
\end{abstract}

\section{Introduction}

With the rapid development of social economy and the increasing demand for electric energy, the problem of security and stability of power grid is becoming more and more prominent [1-2]. Under the condition of power grid interconnection, the destruction of power system stability has more and more influence on power system and social economy [3-4]. If an effective security control strategy can be adopted, it can be targeted to prevent power outages, which is of great practical significance to fully tap the power supply potential of the power grid and ensure the safe, stable and efficient economic operation of the power grid [5].

The prevention and control strategy was first put forward in 1974, and after years of development, it has now formed a more mature system. The system includes the screening of prediction accidents, the establishment of objective function and the requirements of operation planning. The current power system security control strategy is to eliminate or reduce the hidden dangers of power system operation by establishing optimization model and optimization strategy. [6-7].

The most ideal result of the voltage stability preventive control strategy is to control the voltage level of the region purposefully by controlling the parameters of the power system. After the current power system defense line applies this strategy to reduce the impact of the expected accident on the voltage of the whole network, the voltage level needs to be adjusted according to the stability characteristic index. In order to achieve targeted voltage stability level regulation, it is necessary to consider not only the characteristics of voltage stability itself, but also the coupling relationship of the network. [7-8].

Considering that the operating parameters will change continuously in the process of power grid operation, in order to quantitatively track the influence degree of each parameter, it is necessary to grasp the coupling characteristics and stability characteristics of the whole power grid from the macro [9]. For the whole system, the impact of the disturbance on the voltage level of the whole power system determines the anti-disturbance ability of the whole power system [10]. In order to meet the requirements of power grid dispatching and operation, by adjusting the parameters of power system, network structure, load model and other conditions [11]. The stability level under different operating conditions can be enhanced, and the electrical characteristics of complex power grid can be deeply understood [12].

The self-stability characteristics and stabilization characteristics and leading factors of each node can be deeply analysed [13]. The change trend of each disturbance impact after the change of operating conditions can be obtained, and the change law of antidisturbance ability of power grid under different operating conditions can be effectively analyzed. That is to say, the impact size of various disturbance types on the whole network can be obtained. according to the influence degree index, the impact size can be reduced pertinently, which can be used as the global strategy of voltage stability prevention and control, and the influence trend of different strategies on the stability characteristics can be analyzed. It is helpful to deeply understand the electrical characteristics of the network and formulate the corresponding planning measures.

\section{Strategic principle}

A method for obtaining the voltage stability characteristic curve is proposed in reference 13, which is used to study the coupling characteristics of each region of the power system. The typical voltage stability characteristic curve shown in figure 1 is taken as an example to illustrate the practical application of this method.

*orresponding author: huzhen hz@foxmail.com 


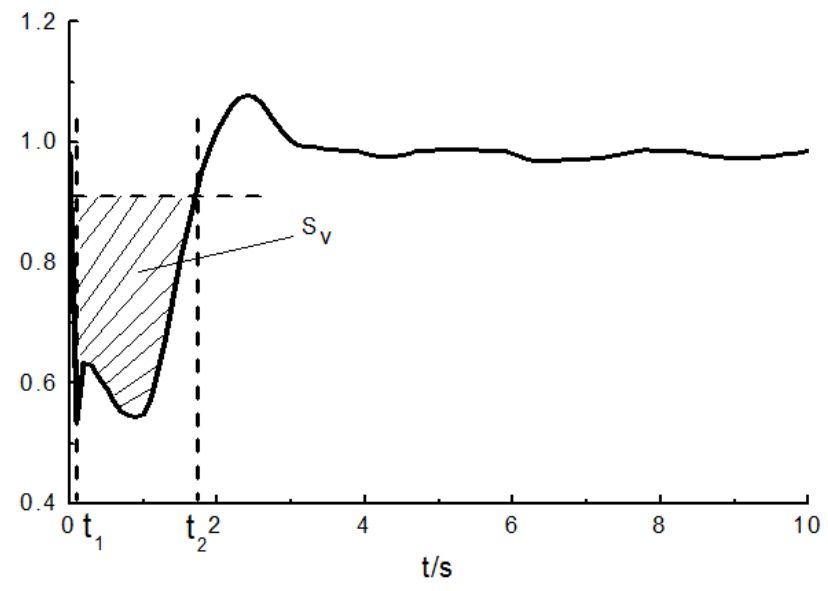

Fig. 1. Voltage stability characteristic diagram

Thus, the voltage stability characteristic index can be defined as follows.

$$
S_{V}(T)=\int_{t_{1}}^{t_{2}}\left|V(t)-V_{0}\right| \mathrm{d} T(1)
$$

Furthermore, the sensitivity index of stability characteristics to control parameters can be obtained.

$$
\Delta S_{\mathrm{Va}}(\mathrm{T})=\frac{S_{V}\left(t_{2}\right)-S_{V}\left(\mathrm{t}_{1}\right)}{\Delta \alpha}(2)
$$

According to formula 2, the sensitivity of the voltage stability level of each region relative to a certain control parameter can be obtained quickly, and then the voltage stability characteristics of each region can be controlled pertinently by adjusting the size of the control parameters. It can be seen that the strategy can also be used to adjust the stability level of a node or region.

\section{Preventive control method}

Based on the coupling characteristic index proposed in reference 13 and the stability margin index in chapter 3 , the objective function of the security and stability preventive control strategy based on characteristic analysis can be obtained.

$$
\Delta \mathrm{C}=\min \sum_{1}^{\mathrm{n}}\left(\mathrm{c}_{S I} S I+\mathrm{c}_{\mathrm{i}} \Delta \mu_{i}\right)(3)
$$

The meaning of sensitivity SI and margin $u$ can be referred to in reference 13 , while $C_{S I}$ and $C_{i}$ are the corresponding cost weight values.

The cost weight value can reflect the cost level of controlling a certain target, according to the dispatching operation experience, Generally, the lowest cost is OLTC tap, reactive power compensation and phase shifter, and so on, while the high cost includes regulating generator output and load shedding and so on.

$$
\left\{\begin{array}{l}
\sum_{j=1}^{n} \mu_{a}\left(\mathrm{~d} \alpha^{+}-\mathrm{d} \alpha^{-}\right) \geq k M_{\mathrm{req}}-M_{i} \\
\sum_{j=1}^{n_{k}}\left(\Delta P^{+}-\Delta P^{-}\right)=0 \\
0 \leq \mathrm{d} \alpha^{+} \leq \alpha^{\max }-\alpha^{0} \\
0 \leq \mathrm{d} \alpha^{+} \leq \alpha^{0}-\alpha^{\min }
\end{array}\right.
$$

Where a represents the control variable, $\mathrm{M}_{\text {req }}$ and $\mathrm{M}_{i}$ represent the voltage stability margin and the minimum threshold, respectively, in the current operating state. The $\mathrm{a}^{\mathrm{max}}$ and $\mathrm{a}^{\mathrm{min}}$ represent the maximum and minimum of the control variable, $\mathrm{a}^{0}$ represents the size of the control variable in the initial running state, and $k$ represents the compensation factor.

It can be seen that the above formula contains the inequality constraint of the objective function, the upper and lower limit requirements of the target variable and the constraint condition of active power balance when the network loss is ignored.

The control variable constraints are shown in the following formula.

$$
\left\{\begin{array}{l}
\Delta t_{j}^{+} \leq \min \left\{k_{t} t_{j, \text { step }}, \quad t_{j, \max }-t_{j, 0}\right\} \\
\Delta t_{j}^{-} \leq \min \left\{k_{t} t_{j, \text { step }}, t_{j, 0}-t_{j, \min }\right\} \\
\Delta V_{g j}^{+} \leq \min \left\{\Delta V_{a d}, \quad \Delta V_{g j, \max }-\Delta V_{g j, 0}\right\} \\
\Delta V_{g j}^{-} \leq \min \left\{\Delta V_{a d}, \quad \Delta V_{g j, 0}-\Delta V_{g j, \min }\right\}
\end{array}\right.
$$

Among them, $\Delta t_{j}^{+}, \Delta t_{j}^{-}, \Delta V_{g j}^{+}$and $\Delta V_{g j}^{-}$represent the positive and negative adjustment difference of branch variables or terminal voltage, $k_{t}$ is the regulation step requirement, $t_{j}$, step is the regulation step requirement, $\Delta V_{a d}$ is the terminal voltage requirement.

The voltage stability preventive control strategy based on characteristic analysis proposed in this chapter includes two aspects. First, through the influence degree of the stability characteristics of each parameter, the influence degree of each variable on the stability characteristics and the leading factors are obtained, and the stability characteristics of a certain region are controlled pertinently. The second is the global strategy, that is, through the influence characteristics between the disturbance events themselves and the whole system, the impact of the disturbance on the stability of the whole network can be weakened by switching reactive power equipment, changing the network structure and adjusting the load.

The essence of voltage stability preventive control strategy based on characteristic analysis is to reduce the impact of disturbance on power grid by using the method of influence degree and stability characteristic index. it can be seen that the strategy method should be tracked online in real time and improved continuously. It is very 
difficult and unnecessary, the system commonly used adjustment methods are also difficult to achieve real-time no-difference smooth adjustment, only need to monitor the target does not exceed the threshold. The regulation method based on the coupling and stability characteristics of power system is to prevent and control the disturbance events in essence, and to analyze its leading factors, which is helpful to fundamentally improve the stability level of power system.

\section{Simulation analysis}

The purpose of the strategy is to improve the voltage stability of the whole network and enhance the antidisturbance ability of the whole power system. The reference power flow (903) can be set by combining the power grid data of a province in central China with the actual planning demand, and the negative load 100MW (903 de-load) can be withdrawn from the power grid data of a province in central China. In the center of the middle load, the $3 *-1.66667$ var parallel capacitance at node 8 (903 decapacitance) and the series connection (903AM) between node 2 AJC and node $9 \mathrm{MF}$ are withdrawn.

The impact comparison under different policies is shown in tables 1 and 2 .

Table 1. Stability influence degree under different Strategies

\begin{tabular}{|c|c|c|c|c|}
\hline Strategy & 903 & $\begin{array}{c}903 \\
\text { de- } \\
\text { load }\end{array}$ & $\begin{array}{c}903 \\
\text { decapacitance }\end{array}$ & $\begin{array}{l}903 \\
\text { AM }\end{array}$ \\
\hline 1 & 1.74 & 1.74 & 1.73 & 1.52 \\
\hline 2 & 2.25 & 2.25 & 2.26 & 1.67 \\
\hline 4 & 1.8 & 1.8 & 1.8 & 1.72 \\
\hline 5 & 0.38 & 0.38 & 0.38 & 0.34 \\
\hline 6 & 0.7 & 0.7 & 0.7 & 0.62 \\
\hline 7 & 1.22 & 1.23 & 1.22 & 1.09 \\
\hline 8 & 4.02 & 4.02 & 4.02 & 4.35 \\
\hline 9 & 2.01 & 2.01 & 2.01 & 1.58 \\
\hline 10 & 2.73 & 2.73 & 2.73 & 2.77 \\
\hline 12 & 0.75 & 0.75 & 0.75 & 0.71 \\
\hline 13 & 0.64 & 0.64 & 0.64 & 0.57 \\
\hline 14 & 1.7 & 0.26 & 0.27 & 0.2 \\
\hline 18 & 0.47 & 1.7 & 1.7 & 1.63 \\
\hline 20 & 0.29 & 0.47 & 0.47 & 0.42 \\
\hline 21 & 0.27 & 0.29 & 0.29 & 0.26 \\
\hline 25 & 0.59 & 0.59 & 0.59 & 0.54 \\
\hline
\end{tabular}

Therefore, the stability influence degree of fault torque can be obtained. As a result, the influence of each different fault condition on the stability and coupling characteristics can be analyzed.

Table 2. Stability influence degree of fault moment

\begin{tabular}{|c|c|c|c|}
\hline \multicolumn{2}{|r|}{903} & \multicolumn{2}{|c|}{$\begin{array}{c}903 \\
\text { de-load }\end{array}$} \\
\hline 8 & 21.5467 & 8 & 21.5426 \\
\hline 2 & 20.1292 & 2 & 20.1571 \\
\hline 10 & 19.9975 & 18 & 19.657 \\
\hline 18 & 19.6337 & 7 & 18.8291 \\
\hline 7 & 18.8151 & 4 & 18.5577 \\
\hline 4 & 18.4351 & 10 & 17.9512 \\
\hline 9 & 15.7451 & 9 & 15.7469 \\
\hline \multicolumn{2}{|c|}{$\begin{array}{c}903 \\
\text { decapacitance }\end{array}$} & \multicolumn{2}{|r|}{$\begin{array}{l}903 \\
\mathrm{AM} \\
\end{array}$} \\
\hline 8 & 21.5444 & 8 & 19.9848 \\
\hline 10 & 19.996 & 2 & 19.5771 \\
\hline 18 & 19.6321 & 18 & 19.3931 \\
\hline 2 & 19.57 & 10 & 19.3692 \\
\hline 7 & 18.8085 & 7 & 18.6498 \\
\hline 4 & 18.5 & 4 & 16.7071 \\
\hline 9 & 15.7512 & 9 & 15.3906 \\
\hline
\end{tabular}

The above table shows the influence degree of power grid stability under different power flow conditions. it can be seen that the influence degree of similar disturbance events has changed. taking Node 8 as an example, The state of power flow with disturbance is closely related to the degree of influence. the main reason why $903 \mathrm{AM}$ is different from the other three methods is that it changes the topology and the power flow has changed greatly. The decrease of influence degree shows that for this kind of disturbance, AM direct connection is beneficial to the improvement of the stability of the whole network, and can effectively reduce the impact of the disturbance on the power grid.

In order to verify the effectiveness of the global strategy proposed in this paper, because the voltage variation of each node is basically the same, the voltage of node 27 at the end of the system is selected as an example. The voltage curves of node 27 after different disturbances under reference power flow and the voltage curves of node 27 with the same disturbance under different power flows are given respectively, as shown in figs. 2 .

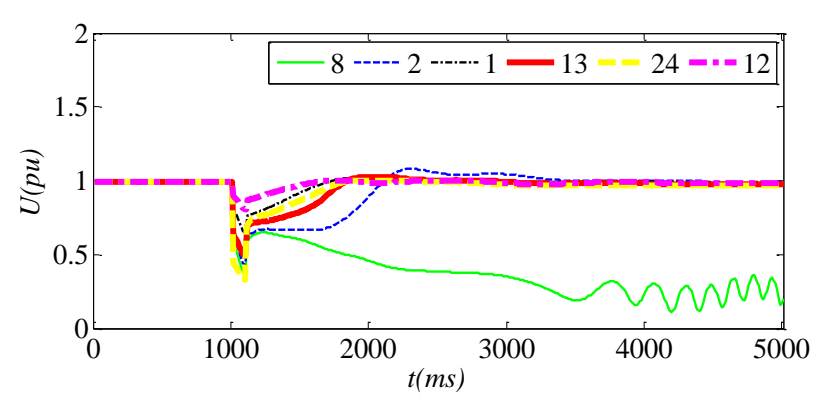

Fig. 2. Voltage at Node 27 after different disturbances 
According to the analysis results of figure 2, it can be seen that node 8 in the disturbance set has the greatest impact on the whole power grid, which needs to be analyzed emphatically. Under the same operating conditions and fault conditions, the stability of the power grid at the fault point will have a greater impact on the whole power system, and then make the voltage unstable. by doing $\mathrm{N} \leqslant 1$ on the whole system and analyzing the power flow, it can be seen that the stability of the power system is greatly affected by the location of the fault point. Under the reference power flow, only node 8 finally makes the voltage unstable, which shows that the results of table 2 analysis are in line with the actual power system.

In the process of operation and dispatching of the actual power network, the security control strategy proposed in this paper can macroscopically reduce the influence of disturbance on the whole system and improve the voltage stability level of the whole network. Through the rapid adjustment of some controllable parameters, we can achieve the purpose of controlling the local regional stability characteristics, so as to make up for the shortcomings of the power system stability characteristics.

When the proposed strategy is applied to the actual power grid, the power of the transmission section in high water area can be increased by nearly 200MW. Assuming that the high water period is 3 months, the electricity price is calculated according to 90 days, and the electricity price is $0.588 \times 10 \sim(-8) \mathrm{kWh}$, the benefit can be increased by 254.016 million yuan, which verifies the feasibility, robustness and reliability of the proposed strategy.

\section{Conclusion}

Based on the characteristic analysis and the constraint conditions of each variable, a voltage stability prevention and control strategy based on characteristic analysis is proposed in this paper, which considers reducing the impact of the expected accident on the stability level from the point of view of the influence degree. By dynamically tracking the change of characteristics, the strategy can be optimized adaptively and follow the change of running state.

Targeted optimization and adjustment of the coupling characteristics of the power grid to achieve the purpose of reducing or even avoiding unstable accidents, we can make full use of the limited funds. Through the proposed strategy, the scheduling and production arrangement can be carried out pertinently, which is helpful for the grid operators to evaluate the power grid accurately. On the premise of ensuring the safe and stable operation of the power grid, we should arrange the investment plan reasonably and obtain greater investment benefit and profit.

\section{Reference}

1. Baghaee, Hamid Reza; Mirsalim, Mojtaba; Gharehpetan, Gevork B.; et al. Nonlinear Load Sharing and Voltage Compensation of Microgrids Based on Harmonic Power-Flow Calculations Using Radial Basis Function Neural Networks. IEEE systems journal. 12, 3 (2018)

2. Hazarika, D. New method for monitoring voltage stability condition of a bus ofan interconnectedpower system using measurements of the bus variables. IET Generation, Transmission \& Distribution. 6, 10 (2012)

3. Wang $\mathrm{Y}$, Wang $\mathrm{C}$, Lin $\mathrm{F}$, et al. Zhao J. Incorporating generator equivalent model into voltage stability analysis. IEEE Trans Power System. 28, 4 (2013)

4. Wang, Qi; Tai, Wei; Tang, Yi; et al. A two-layer game theoretical attack-defense model for a false data injection attack against power systems. International Journal of Electrical Power \& Energy Systems. 104, 2 (2019)

5. Wang Y, Pordanjani IR, Li W. Strategy to minimise the load shedding amount for voltagecollapseprevention. IET Generation, Transmission \& Distribution. 5, 3 (2011)

6. Panteli, Mathaios; Trakas, Dimitris N.; Mancarella, Pierluigi; et al. Boosting the Power Grid Resilience to Extreme Weather Events Using Defensive Islanding. IEEE transactions on smart grid. 7, 6 (2016)

7. Xiang, Yingmeng; Wang, Lingfeng; Zhang, Yichi. Adequacy evaluation of electric power grids considering substation cyber vulnerabilities. International Journal of Electrical Power \& Energy Systems. 96, 3 (2018)

8. Gao B, Morison GK, Kundur, P. Voltage stability evaluation using modalanalysis. IEEE Trans Power System. 7, 4 (1992).

9. Tan, Song; Song, Wen-Zhan; Stewart, Michael; et al. Online Data Integrity Attacks Against Real-Time Electrical Market in Smart Grid. IEEE transactions on smart grid. 9, 1 (2018)

10. Chiang HD, Jumeau RJ. Towards a practical performance index for predictingvoltage collapsein electrical power system. IEEE Trans Power System. 10, 2 (1995)

11. Liao, Weixian; Salinas, Sergio; Li, Ming; et al. Cascading Failure Attacks in the Power System: A Stochastic Game Perspective. IEEE internet of things journal. 4, 6 (2017)

12. Albert R, Albert I, Nakarado G L. Structural vulnerability of the North American power grid. Physical Review E. 69, 2 (2004)

13. Hu, Zhen; Li, Xinran. Method considering the dynamic coupling characteristic in power system for stability assessment. IET Generation, Transmission \& Distribution. 11, 10 (2017) 